\title{
REVISIÓN
}

\section{MÉTODOS CROMATOGRÁFICOS PARA LA DETERMINACIÓN DE ENDOSULFÁN EN ALIMENTOS}

\author{
Jorge Alberto ROMERO HERNÁNDEZ1, Araceli AMAYA CHÁVEZ², \\ María Guadalupe MIRANDA RIVERA ${ }^{2}$ y María Magdalena GARCÍA FABILA ${ }^{2}$ *
}

${ }^{1}$ Facultad de Química, Universidad Autónoma del Estado de México, México

${ }^{2}$ Departamento de Farmacia, Facultad de Química, Universidad Autónoma del Estado de México, México

*Autor para correspondencia: mmgarciaf@uamex.mx

(Recibido enero 2017; aceptado diciembre 2017)

Palabras clave: plaguicidas orgánicos persistentes, extracción y limpieza, metodología analítica

\section{RESUMEN}

Se revisaron las técnicas de extracción, limpieza y cuantificación de endosulfán por métodos cromatográficos en matrices alimentarias como productos lácteos, frutas y vegetales, así como pescados y mariscos, publicados en los últimos veinte años. Independientemente del tipo de matriz, su extracción se realiza principalmente con disolventes orgánicos como hexano, metanol, acetonitrilo y acetona. Para la limpieza, lo más comúnmente empleado fueron cartuchos de sílice, columnas de florisil, o $\mathrm{C}_{18}$. En cuanto a la cuantificación, el método más sensible resultó ser la cromatografía de gases acoplada a espectrofotometría de masas (CG-MS). Los alimentos en los que se reportó la presencia de $\alpha, \beta$ y/o sulfato de endosulfán fueron: productos lácteos y sus derivados con un intervalo de concentraciones de 23.99 a $52.2 \mathrm{ng} / \mathrm{g}$; pescados y mariscos de 1.07 a $49.7 \mathrm{ng} / \mathrm{g}$; y vegetales de 0.5 a $5460.0 \mathrm{ng} / \mathrm{g}$. En leche materna se reportaron concentraciones de 0.4 a $10.7 \mathrm{ng} / \mathrm{mL}$ y en grasa hasta $277.39 \mathrm{ng} / \mathrm{g}$.

Keywords: persistent organic pesticides, extraction and cleaning, analytic methodology

\begin{abstract}
Extraction and cleaning techniques plus chromatographic methods were reviewed for the quantification of endosulfan in food matrices such as dairy products, fruits and vegetables, fish and seafood, published in the last twenty years. Regardless of the type of matrix, the extraction of endosulfan is carried out mainly with organic solvents such as hexane, methanol, acetonitrile and acetone. For cleaning, the most common was the use silica cartridges, florisil columns, or $\mathrm{C}_{18}$. The most sensitive quantification method was gas chromatography coupled to mass spectrometry (GC-MS. Foods where it was reported the presence of $\alpha, \beta$ and/or endosulfan sulfate were dairy products and their derivatives in a range of 23.99 to $52.2 \mathrm{ng} / \mathrm{g}$, in fish and shellfish from 1.07 to 49.7 $\mathrm{ng} / \mathrm{g}$, vegetables from 0.5 to $5460.0 \mathrm{ng} / \mathrm{g}$. In breast milk were from 0.4 to $10.7 \mathrm{ng} / \mathrm{mL}$ and in fat until $277.39 \mathrm{ng} / \mathrm{g}$.
\end{abstract}




\section{INTRODUCCIÓN}

A través de la historia se han utilizado una gran cantidad de plaguicidas para el control de plagas que afectan los cultivos y para el control de vectores de enfermedades (Burgos et al. 2005). Se definen como una sustancia o mezcla de sustancias, cuyo objetivo es prevenir, destruir o mitigar cualquier plaga, ya sean insectos, roedores, hongos, nematodos, algas, macrófitas terrestres o acuáticas, bacterias o virus (Cejudo et al. 2012). De acuerdo a su composición química u origen se clasifican en diferentes grupos, por ejemplo organoclorados, organofosforados, carbamatos, tiocarbamatos, piretroides, organoazufrados, dinitrofenoles, compuestos inorgánicos, compuestos de origen botánico, biológicos, organoestánicos y otros de composición diversa (Castrejón Godínez et al. 2014).

Los plaguicidas organoclorados (POC) son moléculas cíclicas orgánicas sintéticas derivadas de la sustitución de hidrógenos por átomos de cloro. Presentan características lipofílicas con alta persistencia y resistencia a la degradación, con una vida media en humanos cercana a los 10 años (Castilla et al. 2012, Gomes et al. 2013). Los POC son considerados disruptores endocrinos, precursores de carcinogénesis en roedores, causantes de linfoma de non-Hodgkin's; inducen hepatotoxicidad e inmunotoxicidad, además de efectos en el neurocomportamiento (Gomes et al. 2013). Tienen, la capacidad de acumularse en el tejido adiposo de animales y de biomagnificarse a través de la cadena trófica. Debido a lo anterior, en la década de los setenta se estableció a nivel mundial su restricción, principalmente en usos agrícolas y sanitarios, primero en países desarrollados y progresivamente en países en vías de desarrollo (Der 2008, Chacón et al. 2014).

En 2001, 150 países participaron en el convenio de Estocolmo, tratado internacional para el control o eliminación de compuestos orgánicos persistentes (COPs). Se centró la atención en 12 compuestos que coloquialmente han sido nombrados como "la docena sucia", de los cuales nueve son prioritarios para ser eliminados, entre ellos ocho son plaguicidas (aldrin, endrin, dieldrín, toxafeno, mírex, heptacloro, DDT, clordano), un producto de uso industrial (mezcla de bifenilos policlorados) y tres que se reduciría su producción y uso al máximo, hexacloro ciclo benceno (HCB), dioxinas y furanos (UNEP 2009). En la Convención de Estocolmo del 2009, se consideró al endosulfán, así como a sus derivados $\alpha$ y $\beta$-endosulfán (también nombrados I y II endosulfán) y al sulfato de endosulfán dentro de los COPs (UNEP 2009).

Fue producido por primera vez por Hoechtst AG en los 50's, hoy Aventis (Ize 2011), se introdujo como insecticida no sistémico para controlar plagas en semillas, granos, frutas, hortalizas, cultivos de tabaco y algodón. Posteriormente, fue aplicado para el control de vectores de enfermedades como la mosca tse-tse asociada a la tripanosomiasis o enfermedad del sueño, (Joint Canada-Philippines Planning Committee 1995).

Pertenece al grupo de ciclodienos, con identificación química 6,7,8,9,10,10-hexacloro-1,5,5a,6,9,9ahexahidro-6,9-metano-2,4,3-benzodioxatiepin-3-óxido. Es muy soluble en disolventes orgánicos y poco soluble en agua $(<\mathrm{a} 0,5 \mathrm{mg} / \mathrm{L})$, tiene presión de vapor alta que le confiere semivolatilidad, es químicamente estable en aire, favoreciendo su difusión en matrices lipofílicas e hidrofílicas. Sus estereoisómeros $\alpha$ y $\beta$ se presentan en una relación 7:3 (Betancur et al. 2015), con puntos de fusión para cada isómero de $109^{\circ} \mathrm{C}$ y $213^{\circ} \mathrm{C}$, respectivamente y solubilidad en agua de 0.32 y $0.33 \mathrm{mg} / \mathrm{L} \mathrm{a} 22^{\circ} \mathrm{C}$ (Lenardón et al. 2001). Su principal metabolito es el sulfato de endosulfán que se encuentra en el ambiente como resultado de la fotólisis y de la oxidación por microorganismos de sus estereoisómeros $(\alpha$ y $\beta$ ). Otros metabolitos son: endosulfán diol, endosulfán éter, endosulfán lactona, endosulfán hidroxieter y endosulfán ácido carboxílico. Algunos resultan ser más tóxicos y con mayor nivel de bioacumulación (Ize 2011, Betancur et al. 2015). Su concentración total es expresada generalmente como la suma de sus isómeros $\alpha, \beta$ y sulfato de endosulfán ( $\Sigma_{\text {endosulfán})}$ (UNEP 2009). Su tiempo de degradación va de los 123 a 391 días en suelo y para sus estereoisómeros de 25 a 128 días. En agua se ha reportado una vida media de cuatro a 180 días, en condiciones anaeróbicas (Joint Canada-Philippines Planning Committee 1995, Betancur et al. 2015).

Su aplicación ha sido constante en países en vías de desarrollo. Por ejemplo, en México, donde no existe un programa nacional de monitoreo ambiental del uso de plaguicidas, en el 2010 se importaron cerca de 500 toneladas, que se utilizaron en cultivos de alfalfa, algodón, caña de azúcar, cebada, chile, chabacano, ciruelo, durazno, fresa, frijol, jitomate, maíz, trigo, piña, plantas ornamentales y pasto (Ize 2011). Por otra parte, en California, USA donde existe un programa de monitoreo para este tipo de compuestos, se observó que su empleo disminuyó alrededor de una orden de magnitud entre 1993 y 2007, con un promedio anual de 219 a 23 toneladas respectivamente. Su aplicación fue en 72 cultivos entre los que destacan algodón, tomate, lechuga y alfalfa (Silva y Wesley 2010).

Se ha encontrado en suelo superficial en el estado de Chiapas, México, a una concentración de $36.75 \mathrm{mg} / \mathrm{kg}$, valor que sobrepasa lo establecido por la Organización Mundial de la Salud (Escobar et al. 2015). Las 
principales vías de exposición de los organismos son la oral e inhalatoria y en menor medida la dérmica. Los efectos reportados por exposición crónica en animales son: daños en hígado, riñones y sangre, así como disrupción endócrina e inmunosupresión. En humanos los daños a la salud varían desde irritabilidad, inquietud, espasmos musculares, convulsiones, daño renal y/o hepático, hasta la muerte (ATSDR 2013, Betancur et al. 2015).

Los animales y las plantas que están expuestos al suelo y al agua contaminados con COP, pueden metabolizarlos o biomagnificarlos en la cadena alimenticia hasta llegar al ser humano. El consumo de agua, vegetales, lácteos y/o cárnicos contaminados, posicionan a éstos productos como la fuente principal de exposición al endosulfán para el hombre (Chacón et al. 2014). Existe gran interés en la exposición humana, especialmente en el momento más vulnerable que es la niñez. En el cuadro I, se muestran diferentes estudios en alimentos de origen animal, productos lácteos, pescados y mariscos donde se encontró la presencia de $\alpha, \beta$ o sulfato de endosulfán.

\section{ENDOSULFÁN EN ALIMENTOS}

\section{Productos lácteos}

La leche y sus derivados, figuran como los productos de origen animal con mayor importancia en la exposición al endosulfán y sus metabolitos, debido a que constituyen una fuente importante de nutrimentos para el hombre (Chacón et al. 2014). Así mismo, pueden ser indicadores del grado de contaminación ambiental al que está sometida la población (Der 2008). Se ha reportado su presencia en leche materna en concentraciones de 3 a $277.39 \mathrm{ng} / \mathrm{g}$ en base lipídica (Rodas-Ortiz et al. 2008, Freguglia et al. 2011).

En el cuadro I, se observa que la concentración de $\Sigma_{\text {endosulfán en leche bovina se incrementó en casi cinco }}$ veces del año 2003 al 2012, de 0.009 a $0.043 \mathrm{mg} / \mathrm{kg}$, respectivamente, (Lorenzatti et al. 2003, CastillaPinedo et al. 2012). Este aumento de concentración podría deberse al tipo de suplemento alimenticio suministrado a bovinos, ya que se ha observado una mayor contaminación del producto lácteo que deriva de aquellos ejemplares alimentados con plantas expuestas al endosulfán (Hernández et al. 2010).

De acuerdo a las concentraciones de endosulfán en la última década (cuadro I) los productos lácteos que sobrepasaron el límite máximo de residuos (LMR) de $0.01 \mathrm{mg} / \mathrm{kg}$ de grasa de acuerdo al Codex Alimentarius (Fernícola 1985, Gomes et al. 2013) fueron el yogurt, la leche bovina, la leche de cabra y la manteca de leche de vaca (Lorenzatti et al. 2003, Prado et al. 2007, Medina et al. 2010 y Castilla-Pinedo et al. 2012); la ONU marca un LMR en leche de $0.004 \mathrm{mg} / \mathrm{kg}$ en base grasa con esa referencia la leche materna se incluye entre los alimentos que sobrepasan este límite (Campoy et al. 2001, Cerrillo et al. 2005, Rodas-Ortiz et al. 2008, Freguglia et al. 2011). La mayor presencia de sulfato de endosulfán indica una exposición anterior, mientras que una mayor concentración de los isómeros $\alpha$ y $\beta$, refiere una exposición reciente a este plaguicida (Campoy et al. 2001, Cerrillo et al. 2005).

\section{Pescados y mariscos}

Seha evidenciado su acumulación en animales acuáticos (ATSDR 2013), como camarón $(0.01167 \mu \mathrm{g} / \mathrm{kg})$, bagre (Arius melanopus) 31.24 (49.7 ng/g) (NoreñaBarroso et al. 2004) y mojarra (Cichlasoma spp) (Díaz-González et al. 2005).

\section{Frutas, vegetales, semillas y aceites}

Los estudios realizados en alimentos de origen vegetal se agruparon en frutas, vegetales, semillas y aceites, se presentan en el cuadro II.

La fresa es de las frutas en que se reportan las mayores concentraciones de sulfato de endosulfán con un valor máximo de $0.107 \mathrm{mg} / \mathrm{kg}$, (Cuadro II). En el jitomate saladette producido en Oaxaca, México, se encontraron $3.82 \mathrm{mg} / \mathrm{kg}$ de $\alpha$-endosulfán y

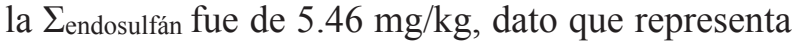
10.9 veces el LMR que es de $0.5 \mathrm{mg} / \mathrm{kg}$ en masa fresca, (Ramírez 2009). Estos resultados muestran que puede haber una acumulación en los vegetales y frutos por la aplicación del plaguicida. En California en los estudios de protección al ambiente y de riesgo alimentario reportaron que se encontró presente de manera residual en 72 productos de consumo humano entre 2001 y 2004, los más importantes fueron algodón, tomate, cabezas de lechuga y alfalfa. Por otra parte, estos estudios consideraron que el brócoli, el melón cantaloupe, las cerezas, el pepino, la pera, los pimientos, y las fresas contienen altos residuos de endosulfán y deben ser considerados como punto de referencia para la protección de la salud (Silva y Wesley 2010).

\section{TÉCNICAS DE EXTRACCIÓN Y LIMPIEZA PARA LA SEPARACIÓN DE ENDOSULFÁN}

Las etapas básicas para la determinación de endosulfán son: extracción, limpieza de la muestra, identificación y cuantificación del plaguicida, 
CUADRO I. ENDOSULFÁN EN ALIMENTOS DE ORIGEN ANIMAL

\begin{tabular}{|c|c|c|c|c|c|c|}
\hline \multicolumn{2}{|c|}{ Matriz } & Metabolito & \multicolumn{3}{|c|}{ Concentración } & Referencia \\
\hline & $\begin{array}{l}\text { Leche de vaca } \\
\text { Manteca }\end{array}$ & $\alpha+\beta$ & \multicolumn{3}{|l|}{$\begin{array}{l}\text { Grasa } \\
0.009 \mathrm{mg} / \mathrm{kg} \\
0.012 \mathrm{mg} / \mathrm{kg}\end{array}$} & Lorenzatti et al. 2003 \\
\hline & Leche de cabra & $\begin{array}{l}\Sigma \\
\alpha \\
\beta\end{array}$ & \multicolumn{3}{|c|}{$\begin{array}{l}\text { Grasa anhidra } \\
52.2 \mathrm{ng} / \mathrm{g} \\
23.99 \pm 56.15 \mathrm{ng} / \mathrm{g} \\
26.51 \pm 19.24 \mathrm{ng} / \mathrm{g}\end{array}$} & Prado et al. 2007 \\
\hline & Leche pasteurizada & $\begin{array}{l}\alpha \\
\beta \\
\text { sulfato }\end{array}$ & \multicolumn{3}{|c|}{$\begin{array}{l}\text { Grasa } \\
0.00004 \mathrm{mg} / \mathrm{g} \\
0.000001 \mathrm{mg} / \mathrm{g} \\
0.000002 \mathrm{mg} / \mathrm{g}\end{array}$} & Castilla-Pinedo et al. 2010 \\
\hline & Leche bovina cruda & endosulfán & \multicolumn{3}{|c|}{$0.35 \pm 0.12 \mu \mathrm{g} / \mathrm{mL}$} & Hernández et al. 2010 \\
\hline & $\begin{array}{l}\text { Leche bovina } \\
\text { pasteurizada }\end{array}$ & $\begin{array}{l}\Sigma \\
\alpha \\
\beta \\
\text { sulfato }\end{array}$ & \multicolumn{3}{|l|}{$\begin{array}{l}\text { Grasa } \\
0.043 \mathrm{mg} / \mathrm{kg} \\
0.04 \mathrm{mg} / \mathrm{kg} \\
0.001 \mathrm{mg} / \mathrm{kg} \\
0.002 \mathrm{mg} / \mathrm{kg}\end{array}$} & Castilla-Pinedo et al. 2012 \\
\hline 递 & Yogurt & endosulfán & \multicolumn{3}{|c|}{$\begin{array}{l}\text { Rango en grasa } \\
0.1105 \text { a } 0.1927 \mathrm{mg} / \mathrm{kg}\end{array}$} & Medina et al. 2010 \\
\hline$\frac{\underline{0}}{0}$ & Mantequilla & endosulfán & \multicolumn{3}{|c|}{$\begin{array}{l}\text { Rango en grasa } \\
0.0012 \text { a } 0.0026 \mathrm{mg} / \mathrm{kg}\end{array}$} & Chacón et al. 2014 \\
\hline & \multirow[t]{5}{*}{ Leche materna } & & $\begin{array}{l}\text { Calostro } \\
(\mathrm{ng} / \mathrm{mL})\end{array}$ & $\begin{array}{l}\text { Leche de } \\
\text { transición } \\
(\mathrm{ng} / \mathrm{mL})\end{array}$ & $\begin{array}{l}\text { Leche } \\
\text { madura } \\
(\mathrm{ng} / \mathrm{mL})\end{array}$ & Campoy et al. 2001 \\
\hline & & $\alpha$ & $<\mathrm{LC}$ & 0.4 & 0.16 a 0.17 & \\
\hline & & $\beta$ & 2.2 a 2.4 & 3.1 a 3.3 & 0.36 a 5.32 & \\
\hline & & endosulfán éter & 0.4 a 3.7 & 1.2 a 2.5 & 0.19 a 7.67 & \\
\hline & & sulfato & 2 a 3.8 & 2.2 a 2.4 & 1.23 & \\
\hline & Leche materna & $\begin{array}{l}\alpha \\
\beta \\
\text { sulfato }\end{array}$ & \multicolumn{3}{|c|}{$\begin{array}{l}0.68 \pm 0.35 \mathrm{ng} / \mathrm{mL} \\
10.70 \pm 8.71 \mathrm{ng} / \mathrm{mL} \\
6.18 \pm 4.18 \mathrm{ng} / \mathrm{mL}\end{array}$} & Cerrillo et al. 2005 \\
\hline & Leche materna & $\beta$ & \multicolumn{3}{|c|}{$\begin{array}{l}\text { Lípidos } \\
277.39 \pm 123.14 \mathrm{ng} / \mathrm{g}\end{array}$} & Rodas-Ortiz et al. 2008 \\
\hline & Leche materna & $\begin{array}{l}\alpha \\
\beta \\
\text { sulfato }\end{array}$ & \multicolumn{3}{|c|}{$\begin{array}{l}\text { No detectados } \\
\text { para } 0.003 \mathrm{ng} / \mu \mathrm{L}\end{array}$} & Freguglia et al. 2011 \\
\hline
\end{tabular}

U.E.: Unidad equivalente, L.C.: Límite cuantificable.

siendo la primera la más crítica del análisis, por ser un factor importante en el porcentaje de recuperación del analito a partir de la matriz. El pre-tratamiento y la limpieza de la muestra en su determinación, se recomienda realizarlos con técnicas avaladas por la USEPA, debido a la confiabilidad y reproducibilidad que con ellas se logra, principalmente la extracción líquido-líquido (LLE) y soxhlet. Adicionalmente se han aplicado técnicas como la extracción en fase sólida (SPE), microextracción en fase sólida (SPME), dispersión de matriz en fase sólida (MSDT), extracción sólido-líquido (SLE) y microextracción en gota (SDME). En el cuadro III, se indican los principales procedimientos de extracción y limpieza en diversas matrices. 
CUADRO I. ENDOSULFÁN EN ALIMENTOS DE ORIGEN ANIMAL

\begin{tabular}{|c|c|c|c|c|}
\hline \multicolumn{2}{|c|}{ Matriz } & \multirow{2}{*}{$\begin{array}{l}\text { Metabolito } \\
\alpha \\
\text { sulfato } \\
\text { endosulfán }\end{array}$} & \multirow{2}{*}{$\begin{array}{l}\text { Concentración } \\
0.00107 \mu \mathrm{g} / \mathrm{L} \\
0.0019 \mathrm{a} 0.1068 \mu \mathrm{g} / \mathrm{L} \\
0.01167 \mu \mathrm{g} / \mathrm{L}\end{array}$} & \multirow{2}{*}{$\begin{array}{l}\text { Referencia } \\
\text { Galindo et al. } 1999\end{array}$} \\
\hline \multirow{5}{*}{ 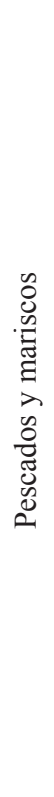 } & $\begin{array}{l}\text { Camarón } \\
\text { (Penaeus vannamei) }\end{array}$ & & & \\
\hline & $\begin{array}{l}\text { Bagre } \\
\text { (Ariopsis assimilis) } \\
\text { (hígado) }\end{array}$ & $\beta$ & $26.57 \mathrm{ng} / \mathrm{g}$ masa seca & Noreña-Barroso et al. 2004 \\
\hline & $\begin{array}{l}\text { Mojarra } \\
\text { (Cichlasoma spp) }\end{array}$ & $\begin{array}{l}\alpha \\
\beta \\
\text { sulfato }\end{array}$ & $\begin{array}{l}\text { masa seca } \\
11.10 \mathrm{a} 99 \mathrm{ng} / \mathrm{g} \\
35.8 \mathrm{ng} / \mathrm{g} \\
49.7 \mathrm{ng} / \mathrm{g}\end{array}$ & Díaz-González et al. 2005 \\
\hline & $\begin{array}{l}\text { Bagre } \\
\text { (Arius melanopus) }\end{array}$ & $\begin{array}{l}\alpha \\
\beta \\
\text { sulfato }\end{array}$ & $\begin{array}{l}\text { promedio en masa seca } \\
10.83 \pm 16.45 \mathrm{ng} / \mathrm{g} \\
2.86 \pm 7.56 \mathrm{ng} / \mathrm{g} \\
31.24 \pm 74.44 \mathrm{ng} / \mathrm{g}\end{array}$ & Díaz-González et al. 2005 \\
\hline & $\begin{array}{l}\text { Ostiones (Cassostrea } \\
\text { virginica, Gmelin y } \\
\text { Cassostrea rhizophorae, } \\
\text { Guilding) } \\
\text { Manchado gar (Lepisos- } \\
\text { teus tropicus Gill) }\end{array}$ & $\Sigma$ & $\begin{array}{l}\text { masa seca } \\
385 \mathrm{pg} / \mathrm{g} \\
26 \mathrm{pg} / \mathrm{g}\end{array}$ & Carvalho et al. 2009 \\
\hline
\end{tabular}

U.E.: Unidad equivalente, L.C.: Límite cuantificable.

CUADRO II. ENDOSULFÁN Y METABOLITOS EN ALIMENTOS DE ORIGEN VEGETAL

\begin{tabular}{|c|c|c|c|c|}
\hline \multicolumn{2}{|c|}{ Matriz } & Metabolito & Concentración & Referencia \\
\hline \multirow{6}{*}{ 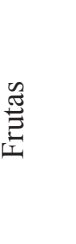 } & Fresas & $\alpha$-endosulfán & 0.005 a $0.019 \mathrm{mg} / \mathrm{kg}$ & Soares et al. 2013 \\
\hline & & $\beta$-endosulfán & 0.005 a $0.032 \mathrm{mg} / \mathrm{kg}$ & \\
\hline & & endosulfán sulfato & 0.013 a $0.107 \mathrm{mg} / \mathrm{kg}$ & \\
\hline & Piña & $\alpha$-endosulfán & $0.0147 \mathrm{mg} / \mathrm{kg}$ exterior & Pinzón et al. 2011 \\
\hline & (Ananas comosus L.) & & $<0.01 \mathrm{mg} / \mathrm{kg}$ interior & \\
\hline & & $\beta$-endosulfán & $0.0613 \mathrm{mg} / \mathrm{kg}$ exterior & \\
\hline \multirow{5}{*}{$\begin{array}{l}\frac{n}{0} \\
\frac{\pi}{0} \\
0 \\
0 \\
0\end{array}$} & Jugo de tomate & $\alpha$-endosulfán & 1 a $5 \mu \mathrm{g} / \mathrm{kg}$ & Albero et al. 2003 \\
\hline & & $\begin{array}{l}\beta \text {-endosulfán } \\
\text { endosulfán sulfato }\end{array}$ & & \\
\hline & Jitomate saladette & $\alpha$-endosulfán & $3.82 \mathrm{mg} / \mathrm{kg}$ & Ramírez 2009 \\
\hline & & इendosulfán & $5.46 \mathrm{mg} / \mathrm{kg}$ & \\
\hline & Calabacita & Eendosulfán & identificado & \\
\hline \multirow{2}{*}{ 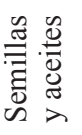 } & Café & $\alpha$-endosulfán & trazas & Dallos y Guerrero 2005 \\
\hline & Aceite de oliva & Sendosulfán & 0.02 a $0.57 \mathrm{mg} / \mathrm{kg}$ & Lentza et al 2001 \\
\hline
\end{tabular}

\section{Extracción Líquido-Líquido (LLE)}

En la extracción líquido-líquido, la muestra en fase acuosa se extrae con un disolvente orgánico inmiscible, se colocan en un embudo de separación junto con sales, ácidos y/o soluciones amortiguadoras, se agitan para aumentar la superficie de contacto entre las dos fases y los líquidos se separan. En la LLE simple, el soluto migra de la fase acuosa a la orgánica, la eficacia de esta extracción depende de las constantes de equilibrio, para el reparto del soluto en 
CUADRO III. PROCEDIMIENTOS DE EXTRACCIÓN Y LIMPIEZA DE ENDOSULFÁN DE DIVERSAS MATRICES

\begin{tabular}{|c|c|c|c|}
\hline Matrices & Extracción & Limpieza & Referencia \\
\hline \multirow[t]{2}{*}{ Leche humana } & \multirow{2}{*}{$\begin{array}{l}\text { LLE: Metanol, oxalato de sodio, éter } \\
\text { etílico/hexanoano }(1: 1 \mathrm{v} / \mathrm{v}), \mathrm{H}_{2} \mathrm{SO}_{4} \\
\text { concentrado, hexano }\end{array}$} & \multirow{2}{*}{$\begin{array}{l}\text { Cartucho de sílice: hexano, elución } \\
\text { hexanoano/ hexano: metanol: isopropa- } \\
\text { nol }(45: 40: 15) \text {, reconstituido en hexano }\end{array}$} & Campoy et al. 2001 \\
\hline & & & Cerrillo et al. 2005 \\
\hline Leche materna & $\begin{array}{l}\text { SLE: } 3 \text { v hexano en mezclador de teji- } \\
\text { do, } \mathrm{Na}_{2} \mathrm{SO}_{4} \text { anhidro }\end{array}$ & $\begin{array}{l}\text { Florisil parcialmente desactivada/ } \\
\mathrm{Na}_{2} \mathrm{SO}_{4} \text { anhidro, elución a hexano, b } \\
\text { hexano:diclorometano }(7: 3)\end{array}$ & Rodas-Ortiz et al. 2008 \\
\hline $\begin{array}{l}\text { Leche humana } \\
\text { Leche en polvo }\end{array}$ & $\begin{array}{l}\text { SPE: florisil calcinado desactivado/ } \\
\text { diclorometano y éter de petróleo }\end{array}$ & & Der 2008 \\
\hline Leche materna & $\begin{array}{l}\text { SPE: } \mathrm{C}_{18}, \text { Metanol, agua destilada, } \\
\text { hexano }\end{array}$ & ـ & Freguglia et al. 2011 \\
\hline Leche bovina & $\begin{array}{l}\text { MSDP: tierras diatomeas/ } \\
\text { hexano:acetona:acetato de etilo( } 4: 2: 1)\end{array}$ & SPE: $\mathrm{C}_{18}$, metanol & Hernández et al. 2010 \\
\hline $\begin{array}{l}\text { Leche pasteuri- } \\
\text { zada }\end{array}$ & $\begin{array}{l}\text { SPE: } \mathrm{C}_{18} \text {, pH } 5-7 \text {, Metanol, metanol } \\
\text { en agua de ósmosis inversa }(2 \%) \text {, } \\
\text { hexano:acetona }(9: 1)\end{array}$ & $\begin{array}{l}\text { Elución con dos volúmenes de } \\
\text { hexano:acetona }(9: 1)\end{array}$ & Castilla-Pinedo et al. 2012 \\
\hline $\begin{array}{l}\text { Leche pasteuri- } \\
\text { zada }\end{array}$ & $\begin{array}{l}\text { SPE: } \mathrm{C}_{18}, \mathrm{pH} 5-7, \text { Metanol, } 2 \mathrm{v} \\
\text { metanol:agua }(2 \%) \text {, hexano:acetona } \\
(90: 10)\end{array}$ & & Castilla-Pinedo et al. 2010 \\
\hline Leche de cabra & $\begin{array}{l}\text { Grasa } \\
\text { Soxhlet: n-hexano (8 h) }\end{array}$ & $\begin{array}{l}\text { C. florisil, hexano: diclorometano ( } 4: 1 \\
\mathrm{v} / \mathrm{v}) \text {, reconstitución con isooctano }\end{array}$ & Prado et al. 2007 \\
\hline Leche cruda & n-hexano & & Ruiz et al. 2008 \\
\hline Yogurt & LLE: Acetonitrilo & Florisil & Medina et al. 2010 \\
\hline Mantequilla & LLE: acetronitrilo & Florisil & Chacón et al. 2014 \\
\hline Grasa porcina & $\begin{array}{l}\text { Columna empacada con alúmina activa- } \\
\text { da neutra, elución hexano. }\end{array}$ & & Rodríguez et al. 2011 \\
\hline Miel & $\begin{array}{l}\text { Pre-tratamiento: dilución metanol, } \mathrm{pH} \\
5 . \\
\text { LLE: acetato de etilo }\end{array}$ & $\begin{array}{l}\mathrm{CCC} \text { : n-hexano, } \mathrm{Na}_{2} \mathrm{SO}_{4} \text { anhidro, sílice } \\
\text { desactivada:florisil }(1: 1), \mathrm{Na}_{2} \mathrm{SO}_{4} \text {, elu- } \\
\text { ción acetato de etilo:n-hexano }(1: 1)\end{array}$ & Rodríguez et al. 2013 \\
\hline Camarón & $\begin{array}{l}\text { Soxhlet: muestra deshidratada, n- } \\
\text { hexano }\end{array}$ & $\begin{array}{l}\text { C. sílice gel/ óxido de aluminio/florisil/ } \\
\mathrm{Na}_{2} \mathrm{SO}_{4} \text { anhidro }(4,4,1,4 \mathrm{~g})\end{array}$ & Galindo et al. 1999 \\
\hline $\begin{array}{l}\text { Bagre } \\
\text { (hígado) }\end{array}$ & Hexano:diclorometano & C. alúmina:sílice & Noreña-Barroso et al. 2004 \\
\hline $\begin{array}{l}\text { Mojarra } \\
\text { Bagre }\end{array}$ & $\begin{array}{l}\text { Soxhlet: muestra deshidratada, } \\
\text { hexano }(8 \mathrm{~h}) \text {, ácido sulfúrico }\end{array}$ & $\begin{array}{l}\text { C. florisil desactivado/ } \mathrm{Na}_{2} \mathrm{SO}_{4} \text { anhidro, } \\
\text { elución hexano, dietiléter-hexano }(25 \% \\
\mathrm{v} / \mathrm{v})\end{array}$ & Díaz-González et al. 2005 \\
\hline $\begin{array}{l}\text { Ostiones } \\
\text { Manchado gar }\end{array}$ & Soxhlet: hexano (8 h) & $\begin{array}{l}\mathrm{H}_{2} \mathrm{SO}_{4}, \mathrm{CC} \text { florisil parcialmente desac- } \\
\text { tivada diclorometano }\left(\mathrm{MeCl}_{2}\right)\end{array}$ & Carvalho et al. 2009 \\
\hline Aceite de oliva & LLE: hexano, acetonitrilo & $\begin{array}{l}\text { Cartucho de alúmina-N con acetoni- } \\
\text { trilo }\end{array}$ & Lentza et al. 2001 \\
\hline
\end{tabular}

LLE: extracción líquido-líquido, SPE: extracción en fase sólida, SPME: micro extracción en fase sólida, PA: poliacrilato, PDMS/DVD: polidimetilsiloxano/ divenilbenceno, MSDP: extracción en dispersión de matriz en fase sólida, SLE: extracción sólido-líquido, SDME: microextracción en gota, CCC: cromatografía clásica en columna, rpm: revoluciones por minuto, min minuto, h: horas, v/v: volumen/ volumen, v: volúmenes, g: gramos, C: columna, CPC: Cromatografía de permeación en gel, M.C.: mini-columna 
CUADRO III. PROCEDIMIENTOS DE EXTRACCIÓN Y LIMPIEZA DE ENDOSULFÁN DE DIVERSAS MATRICES

\begin{tabular}{|c|c|c|c|}
\hline Matrices & Extracción & Limpieza & Referencia \\
\hline Jugo de tomate & $\begin{array}{l}\text { MSDP: florisil, acetona/ doble con ace- } \\
\text { tato de etilo, con baño ultrasónico }\end{array}$ & n-hexano, $\mathrm{Na}_{2} \mathrm{SO}_{4}$ anhidro & Albero et al. 2003 \\
\hline Café & $\begin{array}{l}\text { LLE: } \mathrm{H}_{2} \mathrm{O} \text { :acetona }(2: 1) \text {, acetato de } \\
\text { etilo:ciclohexano }(1: 1)\end{array}$ & $\begin{array}{l}\text { CPC: elución acetato de } \\
\text { etilo:ciclohexano }(1: 1) \\
\text { M.C. sílice gel, elución ciclohexano:ac } \\
\text { etona:acetonitrilo }(86: 10: 4)\end{array}$ & Dallos y Guerrero 2005 \\
\hline Tomates & $\begin{array}{l}\text { LLE: separación de piel y pulpa, acetato } \\
\text { de etilo }\end{array}$ & $\begin{array}{l}\text { C. empacada de } \mathrm{Na}_{2} \mathrm{SO}_{4} \text { anhidro/ } \\
\text { florisil }\end{array}$ & $\begin{array}{l}\text { Gutiérrez y } \\
\text { Londoño } 2009\end{array}$ \\
\hline $\begin{array}{l}\text { Calabacita, } \\
\text { tomate y jito- } \\
\text { mate }\end{array}$ & SLE: acetato de etilo & $\mathrm{Na}_{2} \mathrm{SO}_{4}, \mathrm{NaCl}$ & Ramírez 2009 \\
\hline $\begin{array}{l}\text { Aceites vege- } \\
\text { tales }\end{array}$ & $\begin{array}{l}\text { SPE: Sílice-Hexano } \\
\text { Silica-Acetato de etilo }\end{array}$ & - & Días et al. 2010 \\
\hline Piña & $\begin{array}{l}\text { Pre-tratamiento: acetonitrilo, homogeni- } \\
\text { zación } 18000 \text { rpm por } 2 \text { min, filtración } \\
\text { LLE: hexano, agua grado } 1, \mathrm{Na}_{2} \mathrm{SO}_{4} \\
\text { anhidro }\end{array}$ & $\begin{array}{l}\text { C. empacada de } \mathrm{Na}_{2} \mathrm{SO}_{4} \text { anhidro/ } \\
\text { florisil }\end{array}$ & Pinzón et al. 2011 \\
\hline Fresas & $\begin{array}{l}\text { Pre-tratamiento: } \mathrm{Na}_{2} \mathrm{SO}_{4} \\
\text { Extracción: acetona, diclorometano:n- } \\
\text { hexano }(1: 1)\end{array}$ & Filtración & Soares et al. 2013 \\
\hline $\begin{array}{l}\text { Agua de manan- } \\
\text { tial }\end{array}$ & $\begin{array}{l}\text { SPME: inmersión, fibra PDMS ( } 100 \\
\mu \mathrm{m}), 20 \text { min, } 25^{\circ} \mathrm{C} \text {, baño ultrasónico, } \\
\text { desorción en } \mathrm{CG} \text { a } 220^{\circ} \mathrm{C} \text {, modo split- } \\
\text { less por } 2 \mathrm{~min}\end{array}$ & & Boussahel et al. 2002 \\
\hline $\mathrm{H}_{2} \mathrm{O}$ potable & $\begin{array}{l}\text { SPME: inmersión, fibra PDMS/DVD } \\
(60 \mu \mathrm{m}), 45 \mathrm{~min}, 60^{\circ} \mathrm{C}, 500 \mathrm{rpm} \text {, des- } \\
\text { orción en } \mathrm{CG} \text { a } 250^{\circ} \mathrm{C} \text { por } 5 \mathrm{~min} \text {, modo } \\
\text { splitless por } 2 \mathrm{~min} \text {. }\end{array}$ & 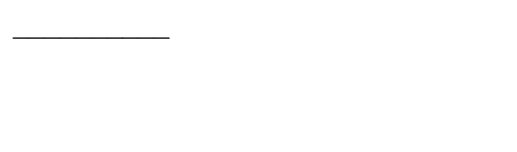 & $\begin{array}{l}\text { Beceiro-González } \\
\text { et al. } 2007\end{array}$ \\
\hline Agua de grifo & $\begin{array}{l}\text { SDME: inmersión, isooctano } 1.5 \mu \mathrm{L}^{-1} \text {, } \\
15 \mathrm{~min}, 800 \mathrm{rpm} \text { a } 22^{\circ} \mathrm{C} \text {, desorción en } \\
\mathrm{CG} 250^{\circ} \mathrm{C}\end{array}$ & + & López-Blanco et al. 2003 \\
\hline
\end{tabular}

LLE: extracción líquido-líquido, SPE: extracción en fase sólida, SPME: micro extracción en fase sólida, PA: poliacrilato, PDMS/DVD: polidimetilsiloxano/ divenilbenceno, MSDP: extracción en dispersión de matriz en fase sólida, SLE: extracción sólido-líquido, SDME: microextracción en gota, CCC: cromatografía clásica en columna, rpm: revoluciones por minuto, min minuto, h: horas, v/v: volumen/ volumen, v: volúmenes, g: gramos, C: columna, CPC: Cromatografía de permeación en gel, M.C.: mini-columna

las dos fases (Nollet 2006). Este tipo de extracción es usualmente seguido por un proceso de limpieza y concentración (Campoy et al. 2001, Cerrillo et al. 2005, Medina et al. 2010), requiere del uso de mucho disolvente y de lavados exhaustivos de material de vidrio y preparación de analistas calificados para tener éxito.

\section{Extracción en fase sólida (SPE)}

En la SPE, el analito previamente disuelto en una fase acuosa se hace pasar a través de un cartucho o membrana donde es atrapado en un material adsorbente y posteriormente es eluído con un poco de disolvente (Kuklenyik et al. 2005, Nollet 2006). Esta es una técnica de pre concentración y limpieza conjugadas, la cual puede ser automatizada. (Cortada 2012).

\section{Extracción Soxhlet}

Es una técnica de separación en matrices sólidas recomendada por EPA, que se basa en la extracción exhaustiva con porciones frescas de disolvente 
orgánico recirculado hacia la muestra por medio de un dedal de vidrio (Canosa 2009). Esta técnica emplea mucho disolvente y tiempo, además requiere de un paso extra de limpieza y concentración (Galindo et al. 1999, Díaz-González et al. 2005, Carvalho et al. 2009).

\section{Dispersión de la matriz en fase sólida (MSDP)}

La MSDP, se fundamenta en la fractura y homogeneización de matrices sólidas o semisólidas. Aumenta el área superficial de las muestras, mezclándolas con un agente dispersante altamente adsorbente y sólido, que posteriormente se transfiere a un cartucho de elución para después realizar su lavado y fraccionamiento con un disolvente adecuado (Canosa 2009), reduciendo los pasos de extracción y limpieza de la muestra a uno solamente. Albero et al. (2003) y Stashenko et al. (2014) logran extraer entre el 25\% y el 32\% más de materia orgánica que con las técnicas Soxhlet y de extracción con disolvente respectivamente.

\section{Extracción sólido-líquido (SLE)}

Según Ramírez (2009), es una técnica recomendada para muestras sólidas. Una porción de muestra homogeneizada se licúa o agita con un disolvente orgánico, la fase orgánica de la separación se deshidrata con sulfato de sodio anhidro $\left(\mathrm{Na}_{2} \mathrm{SO}_{4}\right)$. Es una técnica de extracción sencilla, rápida y con altos porcentajes de recuperación $(\% \mathrm{R})$.

\section{Microextracción en fase sólida (SPME)}

La SPME se basa en el coeficiente de partición de un analito volátil, a través de los procesos de adsorción y desorción hasta llegar al equilibrio termodinámico entre la fase acuosa y el sorbente que esta soportado en una fibra. Esta técnica puede automatizarse y es empleada para el aislamiento de compuestos orgánicos de muestras acuosas o de una fase gaseosa (Alpendurada 2000, Boussahel et al. 2002, Nollet 2006). Además puede realizarse empleando pequeñas cantidades de muestra.

\section{Microextracción en gota (SDME)}

Se basa en el equilibrio entre una gota de disolvente orgánico $(\mu \mathrm{L})$ y la fase acuosa de la muestra, el analito migra a la gota del extractante y es succionada por medio de una microjeringa e inyectada al sistema cromatográfico (López-Blanco et al. 2003). Es una técnica poco empleada por la dificultad que implica mantener una gota de disolvente intacta y recuperarla después (Cortada 2012), la ventaja es usar solo microlitros de disolvente y realizar la inyección casi de inmediato a la extracción.
Comparación de técnicas de extracción y limpieza

Después de la revisión de la diferentes técnicas extractivas para el análisis de endosulfán en diversas matrices, se observó una preferencia en el uso de la SPE para su extracción de leche pasteurizada y leche materna humana, usando cartuchos $\mathrm{C}_{18}$ y combinaciones de eluyentes como metanol, metanol:agua, hexano:acetona (Der 2008, Castilla-Pinedo et al. 2010, 2012, Freguglia et al. 2011). También se empleó la ELL para yogurt y mantequilla, con acetonitrilo como fase orgánica (Campoy et al. 2001, Cerrillo et al. 2005, Medina et al. 2010, Chacón et al. 2014). La extracción Soxhlet con n-hexano, fue preferida para leche de cabra y cruda, al igual que para pescados y mariscos (Galindo et al. 1999, Díaz-González et al. 2005, Prado et al. 2007, Ruiz et al. 2008, Carvalho et al. 2009).

Los procedimientos de limpieza de la muestra, basados en sílice o florisil, fueron aplicados a técnicas de extracción (ELL, Soxhlet, SLE), que consumen volúmenes importantes de disolventes e involucran tiempos largos de procesamiento lo que disminuye la capacidad de analizar grandes cantidades de muestras (Canosa 2009). Otras alternativas de extracción y limpieza son MSDP con tierras diatomeas (Hernández et al. 2010) y la SPE, ambas técnicas disminuyen la cantidad de residuos y los costos. Sin embargo, la SPE tiene la desventaja de presentar variación en los $\% \mathrm{R}$ de los analitos que puede deberse a la presión ejercida en la extracción (Días et al. 2010).

Para la extracción de endosulfán de matrices de origen vegetal, emplearon ELL predominantemente, con disolventes como el hexano y con procesos de limpieza fundamentados en cartuchos o columnas de florisil, alúmina y sulfato de sodio anhidro (Lentza et al. 2001, Dallos y Guerrero 2005, Gutiérrez y Londoño 2009, Pinzón et al. 2011), para muestras como jitomate y calabacita usaron la SLE (Ramírez, 2009). Otra técnica de limpieza evaluada fue con aceite de oliva a través de la remoción de las impureza por precipitación a $-20{ }^{\circ} \mathrm{C}$, método que resultó ser rápido y rentable, según Lentza et al. (2001).

En agua de consumo humano, se han probado técnicas como la SPME y la SDME, que simplifican el proceso de separación, al disminuir los tiempos de partición y eliminar la etapa de limpieza del extracto; se pueden reducir costos y residuos aumentando la efectividad en los límites de detección y reproducibilidad (Cuadro IV) (Alpendurada 2000, Boussahel et al. 2002, López-Blanco et al. 2003, Beceiro-González et al. 2007, Wang et al. 2008). 
CUADRO IV. MÉTODOS DE IDENTIFICACIÓN Y CUANTIFICACIÓN PARA ENDOSULFÁN

\begin{tabular}{|c|c|c|c|c|c|c|c|}
\hline \multirow[b]{2}{*}{$\underset{\sim}{U}$} & \multicolumn{6}{|c|}{ Características analíticas } & \multirow[t]{2}{*}{ Referencia } \\
\hline & Analito & $\% \mathrm{R}$ & $\begin{array}{l}\text { Linealidad } \\
\text { intervalo }\end{array}$ & $r^{2}$ & LD & LC & \\
\hline \multirow{3}{*}{$\sum_{0}^{\infty}$} & $\alpha$ & 93 & $10-160 \mathrm{ng} / \mathrm{L}$ & 0.9967 & \multirow[t]{3}{*}{$11 \mathrm{ng} / \mathrm{L}$} & 32 & \multirow{3}{*}{$\begin{array}{l}\text { Beceiro-González } \\
\text { et al. } 2007\end{array}$} \\
\hline & $\beta$ & 80 & $10-160 \mathrm{ng} / \mathrm{L}$ & 0.9972 & & 32 & \\
\hline & Sulfato & 109 & $5-160$ ng/L & 0.9970 & & $\begin{array}{c}34 \\
\mathrm{ng} / \mathrm{L}\end{array}$ & \\
\hline \multirow{2}{*}{$\sum_{0}^{\infty} \sum_{0}^{\infty}$} & \multirow[t]{2}{*}{$\alpha$} & \multirow[t]{2}{*}{ n.e } & $\begin{array}{c}\text { E.I } \\
0.5-2 \mathrm{ng} / \mu \mathrm{L}\end{array}$ & 0.936 & $0.14 \mu \mathrm{g} / \mathrm{kg}$ & $0.41 \mu \mathrm{g} / \mathrm{kg}$ & \multirow[t]{2}{*}{ Ramirez 2009} \\
\hline & & & $1-10 \mathrm{ng} / \mu \mathrm{L}$ & 1.0 & $\begin{array}{l}0.004 \mu \mathrm{g} / \\
\mathrm{kg}\end{array}$ & $0.01 \mu \mathrm{g} / \mathrm{kg}$ & \\
\hline \multirow{7}{*}{ 导 } & $\alpha$ & n.e & E.I. & 0.957 & $0.005 \mu \mathrm{g} / \mathrm{L}$ & n.e & Boussahel et al. \\
\hline & $\mathrm{E}$ & 92.76 & $\begin{array}{c}\text { P.E } \\
0.003-1 \mathrm{mg} / \mathrm{L}\end{array}$ & 0.9916 & $\begin{array}{c}0.006 \mathrm{mg} / \\
\mathrm{kg}\end{array}$ & n.e & $\begin{array}{l}\text { Medina et al. } \\
2010\end{array}$ \\
\hline & $\alpha$ & $78.06-88.04$ & $2.0-7.0$ & \multirow{3}{*}{$\begin{array}{c}0.9646-0.9927 \\
0.9564-0.9744 \\
0.9390-0.9847\end{array}$} & \multirow{3}{*}{$\begin{array}{c}0.003 \\
0.003 \\
0.007 \\
\mathrm{mg} / \mathrm{kg}\end{array}$} & \multirow{3}{*}{$\begin{array}{c}0.008 \\
0.005 \\
0.013 \\
\mathrm{mg} / \mathrm{kg}\end{array}$} & \multirow[t]{3}{*}{ Soares et al. 2013} \\
\hline & $\beta$ & $81.85-92.20$ & $2.0-17.0$ & & & & \\
\hline & $\mathrm{S}$ & $76.70-89.86$ & $\begin{array}{c}5.0-40.0 \\
\mathrm{ng} / \mathrm{mL}\end{array}$ & & & & \\
\hline & $\mathrm{E}$ & 92.76 & $\begin{array}{c}\text { P. E } \\
0.003-1 \mathrm{mg} / \mathrm{L}\end{array}$ & 0.9916 & $\begin{array}{l}0.006 \mathrm{mg} / \\
\mathrm{kg}\end{array}$ & n.e & $\begin{array}{l}\text { Chacón et al. } \\
2014\end{array}$ \\
\hline & $\begin{array}{l}\alpha \\
\beta \\
S\end{array}$ & n.e & $\begin{array}{c}\text { P. E } \\
20 \mathrm{ng} / \mathrm{mL}\end{array}$ & n.e & $\begin{array}{l}0.001 \mu \mathrm{g} / \\
\mathrm{kg}\end{array}$ & n.e & $\begin{array}{l}\text { Tolentino et al. } \\
2014\end{array}$ \\
\hline 离它 & $\alpha$ & $74.7-74.6$ & $\begin{array}{c}0.037-0.186 \\
\mu \mathrm{g} / \mathrm{mL}\end{array}$ & 0.994 & \multirow[t]{2}{*}{$\begin{array}{l}0.005 \\
\mathrm{mg} / \mathrm{kg}\end{array}$} & \multirow[t]{2}{*}{$\begin{array}{c}0.017 \\
0.015 \\
\mathrm{mg} / \mathrm{kg}\end{array}$} & \multirow[t]{2}{*}{$\begin{array}{l}\text { Dallos y Guerrerc } \\
2005\end{array}$} \\
\hline 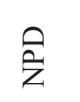 & $\beta$ & $84.8-84.6$ & $\begin{array}{c}0.016-0.080 \\
\mu \mathrm{g} / \mathrm{mL}\end{array}$ & 0.997 & & & \\
\hline 完它 & $\begin{array}{l}\alpha \\
\beta\end{array}$ & $\begin{array}{l}90-100 \\
80-90\end{array}$ & n.e & n.e & n.e & n.e & $\begin{array}{l}\text { Rodríguez et al. } \\
2013\end{array}$ \\
\hline & $\alpha$ & n.e & P. E. & n.e & 0.00127 & 0.00128 & Gutiérrez y \\
\hline 효 & $\beta$ & & $1.5-45$ & & 0.00067 & 0.00067 & Londoño 2009 \\
\hline ৩ & $\mathrm{S}$ & & $\mu \mathrm{g} / \mathrm{kg}$ & & $\begin{array}{c}0.00042 \\
\mathrm{mg} / \mathrm{kg}\end{array}$ & $\begin{array}{l}0.0005 \\
\mathrm{mg} / \mathrm{kg}\end{array}$ & \\
\hline & $\alpha$ & 82 & $0.001-0.2 \mu \mathrm{g} / \mathrm{mL}$ & $>0.99$ & $3 \mathrm{SD}$ de 10 & $0.02 \mathrm{mg} / \mathrm{kg}$ & Lentza et al. \\
\hline ن & $\beta$ & 95 & equivalente & & réplicas & & 2001 \\
\hline & $\mathrm{S}$ & 99 & $0.006-1.2 \mathrm{mg} / \mathrm{kg}$ & & & & \\
\hline
\end{tabular}

SC: sistema cromatográfico, LD: límite de detección, LC: límite de cuantificación, GC-ECD: Cromatografía de gases con detector de captura de electrones, GC-MS: Cromatografía de gases acoplamiento a espectrometría de masas, \%R: porcentaje de recuperación P.E.: patrón externo, E.I.: estándar interno, $\mathrm{r}^{2}$ coeficiente de determinación, FTD: detector termoiónico de flama, GC- $\mu$-ECD: Cromatografía de gases con detector de micro captura de electrones, E: endosulfán, S: endosulfán sulfato, n.e.: no especificado., NPD: detector de nitrógeno y fósforo, SD: desviación estándar. 
CUADRO IV. MÉTODOS DE IDENTIFICACIÓN Y CUANTIFICACIÓN PARA ENDOSULFÁN

\begin{tabular}{|c|c|c|c|c|c|c|c|}
\hline \multirow[b]{2}{*}{ U } & \multicolumn{6}{|c|}{ Características analíticas } & \multirow[t]{2}{*}{ Referencia } \\
\hline & Analito & $\% \mathrm{R}$ & $\begin{array}{l}\text { Linealidad } \\
\text { intervalo }\end{array}$ & $r^{2}$ & LD & $\mathrm{LC}$ & \\
\hline$\bigcup_{\circlearrowright}^{1}$ & $\begin{array}{l}\alpha \\
\beta \\
\mathrm{S}\end{array}$ & $\begin{array}{c}81-91 \\
81-86.7 \\
86.7-100.6\end{array}$ & $0.01-0.10 \mu \mathrm{g} / \mathrm{mL}$ & $\begin{array}{l}0.999 \\
0.998 \\
0.999\end{array}$ & $1 \mu \mathrm{g} / \mathrm{kg}$ & $3 \mu \mathrm{g} / \mathrm{kg}$ & $\begin{array}{l}\text { Albero et al. } \\
2003\end{array}$ \\
\hline 己ِ & $\begin{array}{l}\beta \\
\mathrm{S}\end{array}$ & $93.99-100$ & $\begin{array}{l}\text { E.I } \\
\text { n.e }\end{array}$ & $>0.98$ & n.e & n.e & $\begin{array}{l}\text { Cerrillo et al. } \\
2005\end{array}$ \\
\hline$\bigcup_{\mho}^{\prime} \sum^{\infty}$ & & & & & & & \\
\hline 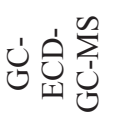 & $\begin{array}{l}\alpha \\
\beta \\
\mathrm{S}\end{array}$ & $>80$ & $\begin{array}{l}\text { P.E } \\
\text { n. e }\end{array}$ & $0.9925-0.9992$ & $\begin{array}{c}0.0001 \\
\mu \mathrm{g} / \mathrm{L}\end{array}$ & $0.02 \mu \mathrm{g} / \mathrm{L}$ & $\begin{array}{l}\text { Castilla-Pinedo } \\
\text { et al. } 2010\end{array}$ \\
\hline$\bigcup_{\circlearrowright}^{1} \bigodot_{\square}^{1} \sum_{\circlearrowright}^{\infty}$ & $\begin{array}{l}\alpha \\
\beta \\
\mathrm{S}\end{array}$ & $\begin{array}{l}95 \\
91 \\
87\end{array}$ & $\begin{array}{c}\text { P. E. } \\
0.01-0.05 \\
\text { ng/ } / \mu \mathrm{L}\end{array}$ & $\begin{array}{l}0.9923 \\
0.9993 \\
0.9989\end{array}$ & $\begin{array}{c}0.003 \mathrm{ng} / \\
\mu \mathrm{L}\end{array}$ & $0.009 \mathrm{ng} / \mu \mathrm{L}$ & $\begin{array}{l}\text { Freguglia et al. } \\
2011\end{array}$ \\
\hline
\end{tabular}

SC: sistema cromatográfico, LD: límite de detección, LC: límite de cuantificación, GC-ECD: Cromatografía de gases con detector de captura de electrones, GC-MS: Cromatografía de gases acoplamiento a espectrometría de masas, \%R: porcentaje de recuperación P.E.: patrón externo, E.I.: estándar interno, $\mathrm{r}^{2:}$ coeficiente de determinación, FTD: detector termoiónico de flama, GC- $\mu$-ECD: Cromatografía de gases con detector de micro captura de electrones, E: endosulfán, S: endosulfán sulfato, n.e.: no especificado., NPD: detector de nitrógeno y fósforo, SD: desviación estándar.

\section{MÉTODOS DE CUANTIFICACIÓN DE ENDOSULFÁN}

Al margen de la inocuidad alimentaria se han desarrollado diversas herramientas analíticas (Rodríguez et al. 2013), con el afán de identificar y cuantificar contaminantes como el endosulfán, mediante cromatografía de gases (GC) acoplada a detectores selectivos (Ramírez 2009), lo que conlleva a la identificación de los isómeros y sus metabolitos, disminuir interferencias de la matriz y mejorar los límites de detección y cuantificación (Betancur et al. 2015).

\section{Cromatografía de gases con detector de captura de electrones (GC-ECD)}

En el cuadro IV, se observa una predominancia de los métodos cromatográficos acoplados a ECD, con variantes como $\mu$-ECD, debido a que responde de forma altamente selectiva frente a compuestos que presenten grupos de elevada afinidad electrónica, como grupos nitro y en particular halógenos como es el caso de la molécula del endosulfán (Soares et al. 2013). En la mayoría de los análisis por GC-ECD, las condiciones de trabajo del inyector van de $220-260^{\circ} \mathrm{C}$ en modo splitless, rampas de temperatura en el horno de la columna alrededor de $90 \pm 10^{\circ} \mathrm{C}$ hasta un máximo de $300^{\circ} \mathrm{C}$ y para el detector de $310 \pm 10^{\circ} \mathrm{C}$. Este método se ha empleado para analizarlo en diversas matrices como frutas, verduras, lácteos y aceites, con \%R que van de 74.6 a 99\% (Galindo et al. 1999, Calderón et al. 2001, Campoy et al. 2001, Díaz-González et al. 2005, Lorenzatti et al. 2006, Nag et al. 2007, Prado et al. 2007, Fernández-Bringas et al. 2008, CastillaPinedo et al. 2010, Hernández et al. 2010, Pinzón et al. 2011), con límites de detección de hasta 0.0001 $\mathrm{mg} / \mathrm{L}$ y de cuantificación de $0.01 \mathrm{mg} / \mathrm{L}$ (Tolentino et al. 2014).

\section{Cromatografía de gases acoplado a espectrometría de masas (GC-MS)}

La GC-MS resulta ser un método de cuantificación de endosulfán y sus metabolitos, ya que presenta fiabilidad a concentraciones traza en alimentos y una alta selectividad al detectar los comportamientos de ionización específica para cada compuesto (Romero et al. 2007). Las condiciones cromatográficas utilizadas fueron para el inyector temperaturas entre $220-270^{\circ} \mathrm{C}$, tanto en modo split como splitless, condiciones 
similares a GC-ECD en la temperatura del horno (Lentza et al. 2001, Albero et al. 2003, Cerrillo et al. 2005, Beceiro-González et al. 2007, Ramírez 2009, Castilla-Pinedo et al. 2010, Freguglia et al. 2011). El acoplamiento de cromatografía de gases con inyector de vaporización de temperatura programable por ionización electrónica, acoplado a espectrometría de masas con selección de ion precursor (GC/PTV/ $\mathrm{EI} / \mathrm{MS}^{2}$ ) desarrollado por Rodríguez et al. (2011) permitió disminuir la degradación del analito en el puerto de inyección a la par que aumentó la sensibilidad mostrada por detectores ECD los cuales junto con MS son los más sensibles.

En el cuadro IV se muestran la exactitud, linealidad, límite de detección (LD) y límite de cuantificación (LQ) de diferentes métodos reportados en los últimos 20 años para el análisis de endosulfán.

La exactitud, descrita como la concordancia entre el resultado obtenido y la concentración adicionada en cada análisis, demostró \% $\mathrm{R}$ comprendidos entre 74.6 y $100.03 \%$ con ELL (Lentza et al. 2001, Cerrillo et al. 2005, Dallos y Guerrero 2005, Medina et al. 2010, Rodríguez et al. 2013, Chacón et al. 2014), para SPE entre 80 y 97\% (Castilla-Pinedo et al. 2010, Freguglia et al. 2011), para MSDP de 80 a 109\% (Albero et al. 2003, Beceiro-González et al. 2007), para SDME de $10 \%$ en un intervalo de 0.1 a $0.9 \mu \mathrm{g} / \mathrm{L}$ para $\alpha$ y $\beta$ endosulfán en muestras de agua para consumo humano (López-Blanco et al. 2003). Métodos combinados mantuvieron de 54.2 a 92.2 de \%R (Rodríguez et al. 2011, Soares et al. 2013). La linealidad, en forma general, la GC-ECD reportó coeficientes de determinación para el endosulfán sulfato de $\mathrm{r}^{2}=0.9390 \mathrm{a} \mathrm{r}^{2}=0.999$ (Lentza et al. 2001, Albero et al. 2003, Cerrillo et al. 2005, Dallos y Guerrero 2005, Castilla-Pinedo et al. 2010, Freguglia et al. 2011, Soares et al. 2013, Chacón et al. 2014). En las cuantificaciones con GC-MS se obtuvieron coeficientes de determinación de $\mathrm{r}^{2}=0.936$ $\mathrm{a} \mathrm{r}^{2}=1.0$ para analizar $\alpha, \beta$ y sulfato de endosulfán, en un intervalo de 5 a $160 \mathrm{ng} / \mathrm{L}$ o bien de 0.01 a $2 \mu \mathrm{g} /$ mL (Albero et al. 2003, Beceiro-González et al. 2007, Ramírez 2009, Freguglia et al. 2011).

Límite de detección (LD) y de cuantificación (LQ), a pesar de las interferencias por el efecto de la matriz, las concentraciones menores detectadas de los isómeros del endosulfán fueron de 0.00042 a $0.007 \mathrm{mg} / \mathrm{kg}$ empleando GC-ECD. (Dallos y Guerrero 2005, Gutiérrez y Londoño 2009, Soares et al. 2013). Para GC-MS el LD fue de 4 a $140 \mathrm{ng} / \mathrm{kg}$ (Albero et al. 2003, BeceiroGonzález et al. 2007, Ramírez 2009). El límite de cuantificación menor obtenido fue de 0.0005 $\mathrm{mg} / \mathrm{kg}$ empleando GC-ECD (Dallos y Guerrero,
2005, Gutiérrez y Londoño 2009, Soares et al. 2013), por GC-MS el LQ se observó de $0.01 \mu \mathrm{g} / \mathrm{kg}$ (Lentza et al. 2001, Albero et al. 2003, Ramírez 2009). La precisión, muy pocas investigaciones la reportaron a través del coeficiente de variación (CV) para las repeticiones de las determinaciones. Los valores encontrados fueron CV de 1.7 a 13.06 (Boussahel et al. 2002, López-Blanco et al. 2003, Lorenzatti et al. 2003, Ramírez, 2009).

\section{CONCLUSIONES}

El endosulfán es un POC, que se ha encontrado en los últimos 20 años en alimentos como lácteos incluyendo leche materna, frutas, vegetales, semillas, pescados y mariscos. Para extraerlo de alimentos que lo contienen, se han empleado técnicas como la extracción líquido-líquido (LLE) y soxhlet (avaladas por USEPA) pero que requieren mucho tiempo y disolventes orgánicos. Se han aplicado técnicas en fase sólida (SPE), microextracción en fase sólida (SPME), dispersión de matriz en fase sólida (MSDT), extracción sólido-líquido (SLE) y microextracción en gota (SDME), con ventajas como la de realizar preconcentraciones y limpieza conjugadas, disminuir la cantidad de muestra requerida y de residuos químicos.

Para la cuantificación del endosulfán y de sus metabolitos, la cromatografía de gases acoplada a detectores selectivos como el de captura de electrones (GC-ECD) o a espectrometría de masas (GC-MS) han sido las más eficientes, adicionalmente la espectrometría de masas con selección de ion precursor $(\mathrm{GC} /$ $\mathrm{PTV} / \mathrm{EI} / \mathrm{MS}-\mathrm{MS}$ ) ha incrementado la sensibilidad en la cuantificación.

Aun cuando sido restringido o prohibido su uso en muchos países, existe la necesidad de hacer la vigilancia de su persistencia en los alimentos como fuente importante de exposición al plaguicida.

\section{AGRADECIMIENTOS}

Agradecemos al proyecto de investigación "Exposición de lactantes a plaguicidas organoclorados a través de la ingestión de leche materna en el Estado de México" clave 3721/2014/CI de la UAEMex por el financiamiento para la realización del presente documento y a la M.E Sara Ananny Iturbe Peñaloza por la revisión del inglés. Los autores agradecen el financiamiento recibido del CONACyT a través del proyecto de la Red Temática de Plaguicidas con número 280045. 


\section{REFERENCIAS}

ATSDR (2013) Toxicological profile for endosulfan. Agency for Toxic Substances and Disease Registry. Reseña. Atlanta, GA., EUA, 330 pp. https://www.atsdr. cdc.gov/toxprofiles/tp41.pdf

Albero B., Sánchez C. y Tadeo J. (2003). Determination of $\alpha$-endosulfan isomers and endosulfan sulfate in tomato juice by matrix solid-phase dispersion and gas chromatography. J. Chromatograf. A. 1007, 137-143. DOI: 10.1016/S0021-9673(03)00954-3

Alpendurada M. (2000). Solid-phase microextraction: A promising technique for sample preparation in environmental analysis. J. Chromatograf. A. 889, 3-14. DOI 10.1515/sampre-2015-0005

Beceiro-González E., Concha-Graña E., Guimaraes A., Goncalves C., Muniategui-Lorenzo S. y Alpendurada M. (2007). Optimisation and validation of a solid phase microextraction method for simultaneous determination of different types of pesticides in water by gas chromatography - mass spectrometry. J. Chromatograf. A. 1141, 165-173. DOI: 10.1016/j.chroma.2006.12.042

Betancur L.A., Ocampo R. y Ríos L.A. (2015). La problemática del endosulfan: aspectos químicos, analíticos y ambientales. Revista Luna Azul, 40, 293-313. DOI: 10.17151/luaz.2014.40.19 Boussahel R., Bouland S., Moussaoui K., Baudu M. y Montiel A. (2002). Determination of chlorinated pesticides in water by SPME/GC. Water Res. 36, 19091911. https://doi.org/10.1016/S0043-1354(01)00372-4

Burgos A., García C., Aldana M. y Meza M. (2005). Detection and quantification of insecticides in shrimp grown in a coastal farm in Sonora, Mexico. Bull. Environ. Contam. Toxicol. 74, 335-341.

DOI: $10.1007 / \mathrm{s} 00128-004-0589-4$.

Calderón H., González R. y Durán de Bazúa, C. (2001). Plaguicidas organoclorados en sedimentos y organismos acuáticos del lago de Catemaco, Veracruz, México. Rev. Int. Contam. Ambie. 17 (1), 23-30. http:// www.revistascca.unam.mx/rica/index.php/rica/article/ viewFile/25533/24078

Campoy C., Jiménez M., Olea M., Moreno Cañabate F., Oleae N., Bayés R. y Molina J. (2001). Analysis of organochlorine pesticides in human milk: preliminary results. Early Hum. Develop. 65, S183-S190. DOI: $10.1016 / \mathrm{S} 0378-3782(01) 00221-3$

Canosa M. (2009). Desarrollo de metodología analítica para la determinación de triclosán y parabenes, aplicación al estudio de su distribución y transformación en muestras ambientales. Tesis de Doctorado. Santiago de Compostela: Universidad. España, 290 pp.

Castrejón Godínez M.L, Sánchez-Salinas E., Ortiz Hernández M.L. (2014) Plaguicidas: Generalidades, usos e impactos sobre el ambiente y la salud. En: Los plaguicidas en México. Aspectos generales, toxicológicos y Ambientales. (M.L. Ortiz, E. Sánchez, J.L. Folch, A. Olvera, E. Datan) 1a edición 2014. Universidad Autónoma del Estado de Morelos. México 11-35.

Castilla-Pinedo Y., Alvis-Estrada L. y Alvis-Guzmán N. (2010). Exposición a organoclorados por ingesta de leche pasteurizada comercializada en Cartagena, Colombia. Rev. Salud Publica (Bogotá). 12 (1), 14-26. PMID: 20628696

Castilla-Pinedo Y., Mercado I. y González G. (2012). Determinación y cuantificación de los niveles de compuestos organoclorados en leche pasteurizada. Rev. Prod. Lim. 7 (1), 19-31. https://dialnet.unirioja. es/servlet/articulo?codigo $=4331514$

Carvalho F., Villeneuve, J., Cattini C., Rendón J., Mota de Oliveira, J. (2009). Pesticide and PCB residues in the aquatic ecosystems of Laguna de Términos, a protected area of Campeche, Mexico. Chemosphere. 74, 988-995. doi:10.1016/j.chemosphere.2008.09.092

Cejudo E., Meza M., Balderas C., Mondaca H., Rodríguez, R., Renteria M. y Félix A. (2012). Exposición a Plaguicidas organoclorados en niños indígenas de Potam, Sonora, México. Ra Ximhai. 8(2), 121-127. http://oai.redalyc.org/articulo.oa?id=46123333012.

Cerrillo I., Granada A., López M., Olmos B., Jiménez M., Caño A., Olea N. y Olea M. (2005). Endosulfan and its metabolites in fertile women, placenta, cord blood, and human milk. Environ. Res. 98, 233-239.

DOI: 10.1016/j.envres.2004.08.008

Chacón B., Izquierdo P., Allara M., Sánchez E., García A. y Torres G. (2014). Residuos de insecticidas organoclorados en mantequilla de cuatro marcas comerciales elaboradas en Venezuela. Rev. Cient. 24(5), 399-407. http://www.redalyc.org/articulo. oa? $\mathrm{id}=95932260003>$

Cortada C. (2012). Nuevas metodologías y aplicaciones de las técnicas de microextracción líquido-líquido para la determinación de contaminantes orgánicos. Tesis Doctoral. Facultad de Ciencias. Universidad de Alicante. Alicante España. 271pp.

Dallos D. y Guerrero J. (2005). Desarrollo y validación de una metodología para la determinación de plaguicidas en café verde por cromatografía de gases. Revista Colombiana de Química. 34(2), 175-188.

Der S. (2008). Plaguicidas organoclorados en leche materna. Rev. Hosp. Mat. Infa. Ramón Sardá. 27(2), 70-78. https://revistas.unal.edu.co/index.php/rcolquim/ article/view/818

Dias E., Vilela E. y Lancas F. (2010). Análise de pesticidas organoclorados ém óleos vegetais por cromatografía gasosa de alta resoluca (HRGC). Eclé. Quím. 35 (3), 189-193. DOI:10.1590/S0100-46702010000300019 
Díaz-González, Botello A. y Ponce-Vélez G. (2005). Plaguicidas organoclorados en pastos y peces de los sistemas Candelaria-Panlau y Palizada del Este, Laguna de Términos, Campeche, México. En: Golfo de México. Contaminación e impacto ambiental: diagnóstico y tendencias (A Botello A., Rendón-von Osten J., GoldBouchot G. y Agraz-Hernández C. Eds.). 2a . Edición. Univ. Autón. De Campeche, Univ. Nal. Autón. De México, Instuto Nacional de Ecología. pp. 207-224 pp.

Escobar D., Rendón J., Guillén H., Caballero A. y Figueroa J. (2015). Determinación de Hexaclorociclohexano y endosulfán en suelos agrícolas del estado de Chiapas. Revista AIDIS. 8 (2), 161-170. www.revistas.unam. $\mathrm{mx} /$ index.php/aidis/article/download/51635/46105

Fernícola N. (1985). Toxicología de los insecticidas organoclorados. Bol. Ofic. San. Panam. 98 (1), 10-18.

Fernández-Bringas L., Ponce-Vélez G., Laura G., SalgadoUgarte I., Botello, A. y Díaz G. (2008). Organochlorine pesticides in lacustrine sediments and tilapias of Metztitlan, Hidalgo, México. Rev. Biol. Trop. 56 (3), 1381-1390. http://www.scielo.sa.cr/pdf/rbt/v56n3/ art31v56n3.pdf

Freguglia R., Moura G. y Tornisielo L. (2011). Determination of organochlorine pesticide residues in human milk in Piracicaba, SP. Eclé. Quím. 36 (4), 21-27.

Galindo G., Villagrana C. y Lazcano G. (1999). Environmental conditions and pesticide pollution of two coastal ecosystems in the Gulf of California, Mexico. Ecotox. Environ. Saf. 44, 280-286.

DOI: 10.1006/eesa.1999.1836

Gomes J., Amaya A., Waliszewski S., Colín A. y García M. (2013). Extraction and clean-up methods for organochlorine pesticide. Chemosphere 92, 233-246. DOI: 10.1016/j.chemosphere.2013.04.008

Gutiérrez J. y Londoño A. (2009). Determinación de plaguicidas organoclorados y organofosforados en tomates de mercados de cadena en las ciudades de Pereira y Armenia, Colombia. Bol. Latin. Car. Plan. Medi. Arom. 8 (3), 165-171. www.redalyc.org/ pdf $/ 856 / 85611774005$.pdf

Hernández M., Vidal J. y Marrugo J. (2010). Plaguicidas organoclorados en leche de bovinos suplementados con residuos de algodón en San Pedro, Colombia. Rev. Salud Púb. 12 (6), 282-289. www.redalyc.org/ pdf/422/42219912009.pdf

Ize I. (2011). Diagnóstico de la situación del endosulfán en México. Instituto Nacional de Ecología. México. 53 pp. http://www.inecc.gob.mx/descargas/sqre/2011_ diag_endosulfan_mex.pdf

Joint Canada-Philippines Planning Committee. (1995). Meeting Back-ground Report for the International Experts Meeting on Persiten, Organic Pollutants: Towards Global Action, Vancouver, Canada, June 4-8.
Kuklenyik Z., Ye X., Needham L. y Calafat A. (2005). Automated on-line column-switching HPLC-MS/MS method with peak focusing for the determination of nine environmental phenols in urine. Anal. Chem. 7, 5407-5413. DOI: $10.1021 / \mathrm{ac} 050390 \mathrm{~d}$

Lenardón A., De la Sierra P. y Marino F. (2001). Persistencia del endosulfán en medio acuoso estático. Rev. Ecotox. Meio. Amb. 11, 115-126. http://revistas.ufpr. br/pesticidas/article/viewFile/3140/2513

Lentza Ch., Avramidesa E. y Visi E. (2001). Determination of residues of endosulfán and five pyrethroid insecticides in virgin olive oil using gas chromatography with electron-capture detection. J. Chromatograf. A. 921, 297-304. DOI: 10.1016/S0021-9673(01)00874-3

López-Blanco M., Blanco-Cid S., Camacho-Grande B. y Simal-Gándara J. (2003). Aplication of single - drop microextration and comparison with solid -phase microextraction and solid -phase extraction for determination of $\alpha$ - and $\beta$-en dosulfan in water samples by gas chromatography-electron-capture detection. Jour of Chromat A. 984, 245-252.

DOI: 10.1016/S0021-9673(02)01873-3

Lorenzatti E., Maitre M y Lenardón A. (2003). Evaluación de la contaminación con plaguicidas en productos lácteos. Rev. FAVE - Cien Veter. 2 (1), 49-56. https:// bibliotecavirtual.unl.edu.ar/ojs/index.php/FAVEveterinaria/article/.../2205

Lorenzatti E., de la Sierra P., Marino F. y Lenardón A. (2006). Acumulación y persistencia del insecticida endosulfán en soja, como posible factor de contaminación ambiental y alimentaria. FABICIB. 10, 101-111. DOI: 10.14409/fabicib.v10i1.797

Medina C., Allara M., Izquierdo P., Sánchez E., Piñero M. y Torres G. (2010). Residuos de insecticidas organoclorados en yogurt firme en tres marcas comerciales, elaborado en Venezuela. Rev. Cient. FCV-LUZ. 20 (2), 203-211. www.scielo.org.ve/scielo.php?script $=$ sci arttext\&pid...22592010000200014

Nag S., Mahanta S., Raikwar M. y Bhadoria B. (2007). Residues in milk and production performance of goats following the intake of a pesticide (endosulfan). Small Rum. Res. 67, 235-242. DOI: 10.1016/j.smallrumres.2005.10.008

Nollet L. (2006). Cromatographic análisis of the enviroment. CRC Taylor\&Francis Group. 3rd. Edition. United States of America. pp. 41, 46-51.

DOI: $10.1021 / \mathrm{ja} 069720 \mathrm{~h}$

Noreña-Barroso E., Simá-Alvarez R., Gold-Bouchot G. y Zapata-Pérez O. (2004). Persistent organic pollutants and histological lesions in Mayan catfish Ariopsis assimilis from the Bay of Chetumal, Mexico. Mar. Poll. Bull. 48, 263-269.

DOI: 10.1016/j.marpolbul.2003.08.001 
Pinzón M., Londoño A., Blach D., Gutiérrez J. y Rojas A. (2011). Determinación de residuos de plaguicidas organoclorados POR gc- $\mu \mathrm{ECD}$ en frutos de PIÑA (Ananas comosus L.) variedad Golden MD2 en el departamento del Quindío. Rev. Fac. Cien. Bás. 9(2), 4-8. http://www.unipamplona.edu.co/unipamplona/ portalIG/home_10/recursos/general/pag_contenido/ publicaciones/bistua_revista_ciencias_basica/2011/ vol2/13122011/pinzon_magda_ivonne.pdf

Prado G., Carabias R., Rodríguez E. y Herrero E. (2002). Presencia de residuos y contaminantes en leche humana. Revista Española de Salud Pública. 76 (2), 133-147. scielo.isciii.es/scielo.php?script=sci_arttex t\&pid=S1135-57272002000200007

Prado G., Díaz G., Gutiérrez R, Vega y León S., Noa M. y Chávez E. (2007). Residuos de plaguicidas organoclorados en leche de cabra de Querétaro, Querétaro, México. Veter. Méx. 38 (3), 291-301. http://www. redalyc.org/articulo.oa?id $=42338304>$

Ramírez L. (2009). Determinación de pesticidas en vegetales mediante cromatografía de gases-espectrometría de masa/masa (GC-MS/MS). Tesis Ingeniería. Universidad Tecnológica de la Mixteca. México, 92 pp. http:// jupiter.utm.mx/ tesis_dig/10970.pdf

Rodas-Ortíz J., Ceja-Moreno V., González-Navarrete L., Alvarado-Mejía J., Rodríguez-Hernández M. y GoldBouchot G. (2008). Organochlorine pesticides and polychlorinated biphenyls levels in human milk from Chelem, Yucatán, México. Bull. Environ. Contam. Toxicol. 80, 255-259. DOI 10.1007/s00128-007-9356-7

Rodríguez D., Díaz A., Ahumada D. y Guerrero J. (2013). Desarrollo y optimización de una metodología multiresiduo por método simplex para el análisis de plaguicidas en miel de abejas. Rev. Colom. Quím, 42 (1), 1-9. DOI: 10.15446/rev.colomb.quim

Rodríguez O., Soto S., Velázquez L., Rodríguez G. (2011). Desarrollo y validación de un método analítico para la detección y cuantificación de plaguicidas organoclorados en grasa de ganado porcino con un sistema GC/PTV/EI/MS ${ }^{2}$. Vet. México. 42 (2), 101-113. http:// www.redalyc.org/articulo.oa?id=42329683001
Romero R., Fernández J., Plaza P., Garrido A y Martínez J. (2007). Empleo de la espectrometría de masas como herramienta para la determinación de tóxicos en alimentos: Hacia la seguridad alimentaria. Rev. Esp. Salud Púb. 81, 461-474. http://antioquiacolombiawww. redalyc.org/articulo.oa?id=17081504>

Ruiz, Wierna N. y Bovi, G. (2008). Plaguicidas organoclorados en leche cruda comercializada en Jujuy (Argentina). Rev. Toxicol. 25, 61-66. http://antioquiacolombiawww.redalyc.org/articulo.oa?id=91925310>

Silva M. y Wesley C. (2010). Human healt risk assessment of endosulfan: II. Diarity exposure assessment. Regul. Toxicol. Pharma. 56, 18-27. DOI: 10.1016/j.yrtph.2009.08.015

Soares A., Amaral E., Sousa L., Souza S. y Junqueira R. (2013). In-house method validation and ocurrence of alfa-, beta-endosulfan, endosulfán sulphate, lambdacyhalothrin, procymidone and trifluralin residues in strawberry. Cién. Tecnol. Alim, 33 (4), 765-775. DOI: 10.1590/S0101-20612013000400024

Stashenko E., Martínez J.R., y Castrillón J. 2014. Aplicación del método de dispersión de matriz en fase sólida al aislamiento de hidrocarburos de rocas bituminosas. Boletín de Geología, 36(1): 29-35. http://www.scielo.org.co/scielo.php?script=sci_arttext\&pid=S012002832014000100002\&lng=en\&nrm=iso

Tolentino R.G., León, S.V., Bermúdez B.S., Flores G.P., De Lourdes Ramírez Vega M., Vázquez C.R., Vázquez M.R. y Francisca M.V. (2014). Organochlorine pesticides in infant milk formulas marketed in the South of Mexico City. Food. Nutri. Sci. 5, 1290-1298. DOI:10.4236/fns.2014.513140

UNEP. (2009). Endosulfan, report of the persistent organic pollutants Review Committee on the work of its fifth meeting. United Nations Environmental Program. Reporte. Ginebra, Suiza, 26 pp. http://chm.pops.int/ Default.aspx?tabid $=592$

Wang X., Xu, O., Kang C., Cong Y. y Yao D. (2008). The historical residue trends of DDT, hexachlorocyclohexanes and polycyclic aromatic hydrocarbons in an ice core from Mt. Everest, central Himalayas, China. Atmos. Environ. 42, 6699-6709. DOI: 10.1016/j.atmosenv.2008.04.035 